\title{
Emotion Detection Using EEG and ECG Signals from Wearable Textile Devices for Elderly People
}

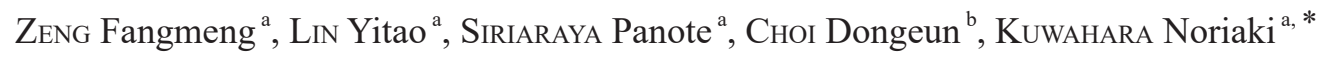 \\ ${ }^{a}$ Graduate School of Science and Technology, Kyoto Institute of Technology, 1 Hashigami-cho, Matsugasaki, Sakyo-ku, Kyoto 606-8585, Japan \\ ${ }^{\mathrm{b}}$ Faculty of Informatics, The University of Fukuchiyama, 3370 Hori, Fukuchiyama, Kyoto 620-0886, Japan
}

Received 30 April 2020; accepted for publication 11 November 2020

\begin{abstract}
The global population is ageing; exacerbating a range of age-related health problems, like dementia. In the late stage of dementia, patients often are unable to find words to express their feelings; causing serious challenges in healthcare. Our aim is to detect the emotions of elderly patients using physiological signals - electroencephalogram (EEG) and electrocardiogram (ECG) - using deep learning neural networks. However, most EEG and ECG monitoring devices are uncomfortable and not suitable for daily wear by elderly people. For this study, a prior experiment was conducted on 5 healthy elderly subjects for binary classification of positive and negative emotions: EEG and ECG data were collected from the subjects, using our own designed wearable textile devices while they watch selected stimuli. We propose an end-to-end deep learning method - Long short-term memory (LSTM) - to detect emotion from raw clean signals after removing noises and baseline wander. LSTM can learn features from raw data directly and achieve binary emotion classification with an accuracy of $76.67 \%$ with EEG signals, $75.00 \%$ with ECG signals, and $95.00 \%$ with EEG and ECG signals, respectively. This proposed system for detecting emotion by deep learning method using our userfriendly and easy-to-wear textile devices offer great prospects for use in everyday care situations and dementia care.
\end{abstract}

Key Words : Emotion, ECG, EEG, Elderly people, Wearable textile devices

\section{Introduction}

The United States and Japan have been facing the ageing problem since the 1980s [1]. This phenomenon has expanded worldwide: In some Asian countries, such as China, India, and Indonesia, the proportion of the ageing people is estimated to more than triple between 2000 and 2050 [2]. Meanwhile, Japan has remained the fastest ageing country in the world. On one hand, this reflects the benefits, such as longevity, reaped from improved living conditions and healthcare systems [3]: Japan has the highest life expectancy at birth worldwide [4]. On the other hand, it highlights the negative impact of marital trends, such as late or non-marriages, on fertility rates - which have been rapidly declining since the brief post World War II baby boom (1947-1949) [5] in Japan. Consequently, the age demographics in Japan have shifted significantly. With elderly population already outnumbering children by 1997 (Statistical Handbook of Japan 2020), younger generations are shouldering a heavier financial and psychological burden when it comes to caring for the elderly. This brings serious challenges to healthcare and social services.

Ageing research is a major theme in Japanese academic universities and industries, including topics on the mechanism of aging, detection of disease, and healthcare. Ageing, in general, brings progressive and generalized impairment of functions [6], affecting the central and peripheral autonomic nervous system, and the regulation of brain activity, heart rate, blood pressure, and temperature [7]. Previous studies [8,9] have indicated that there are several differences between the central nervous system (CNS) in young organisms and old organisms. The electroencephalogram (EEG) known as brain waves directly generated by the human CNS is typically used to pick up changes in activity due to ageing [10, 11]. EEG signals are also closely associated with the generation of emotions, exhibiting different prominent frequency bands at different emotional states. Meanwhile, there are significant agerelated changes in the autonomic nervous system (ANS) which can influence heart rate responses [12]. The electrocardiogram (ECG) signal, an activity of the ANS, will also differ as people age [13]. The heart rate (HR) and heart rate variability (HRV), or variances in time between two successive heartbeats, are common ECG features used for emotion recognition. The significant age-related changes in the CNS and ANS of humans can impair an elderly person's ability to adapt to the stresses and stimuli of everyday life. Ageing is also

* Corresponding author: E-mail : nkuwahar@kit.ac.jp, Tel : +81-75-724-7494, Fax : +81-75-724-7494 
a major risk factor for neurodegenerative diseases such as dementia (a non-curable disease), which is arguably becoming the world's biggest health challenge [14]. In the late stage of dementia, the patients lose the ability to speak; preventing them from expressing their feelings to the caregivers. Our research aim is to detect the emotions of elderly people using physiological signals - EEG and ECG [15] - to improve the quality of care between care receivers and caregivers; emotions are fundamental to human life [16]. Yet, despite advancements in affective computing, and our ability to use physiological signals to accurately recognize emotions, elderly people are often averse to the traditional monitoring devices, such as gel sensors or bulky robotics, which cause stress and inconvenience to both the experimenters and subjects. In our previous study [17], we developed wearable devices made with textiles to measure EEG and ECG signals of elderly people. The devices were designed to be user-friendly, comfortable and suitable for long-term and everyday wear.

Many existing studies focusing on emotion classification using machine learning with physiological signals achieved good results. Atkinson et al. [18] classified EEG-based features into high/ low arousal and positive/negative valence using support vector machine (SVM) through a Database for Emotion Analysis Using Physiological Signals (DEAP) dataset. Jadhav et al. [19] used EEG spectrogram images to detect four emotions (happy, angry, relax, and sad) using K-nearest neighborhood (KNN) through a DEAP dataset. Soleymani et al. [20] applied a SVM classifier for emotion classification for arousal and valence using EEG and eye gaze data on a MAHNOB-HCI database. Guo et al. [21] used a SVM for the classification of positive and negative emotional states using the extracted time domain and frequency domain features from ECG on 25 subjects. Valenza et al. [22] performed valence classification by several classifiers (SVM, KNN, Multi-Layer Perceptron (MLP), etc.) using ECG features on 32 subjects. Kim et al. [23] recognized four emotions (sadness, stress, anger and surprise) by adopting SVM through ECG on 3 males. However, these previous studies share several common features: (1) most of the used classifiers require complex and handcrafted features extraction and feature optimization; (2) only EEG signal or ECG signal was adopted for emotion classification; and (3) it is difficult to assess the performance of their classification results due to the use of different datasets.

Unlike most proposed methods, recurrent neural network (RNN) does not require any feature extraction, which can feed the timeseries data such as physiological signals directly and can learn the nonlinear characteristics of sequential data. RNN takes sequence data as input, loops the evolution direction of the sequence, and connects all nodes by a chain, However, as RNN is trained by backpropagation through time (BPTT), it suffers from the problem of gradient vanishing or gradient explosion when learning long sequences in long term training from traditional RNN: The long term dependency problem of RNN (Sepp Hochreiter, 1991). While long short-term memory networks (LSTM) are a variant of RNN, which were proposed by Hochreiter and Schmidhuber in 1997 [24] to address the long-term dependencies problem of traditional RNN. LSTM networks introduce a new structure called a memory cell or LSTM cell, and the concept of a gating structure and cell state. Each LSTM cell consists of three gates: (1) input gate which acts on input information and determines which values will be updated; (2) forget gate which acts on previous memory information and determines what information to throw away from the cell state; (3) the output gate which acts on output information and determines the update of the final output. Fig. 1 [24] shows the basic structure of LSTM. These gates allow the cells to keep and access information over long periods of time. Due to its unique design structure, LSTM offers a wide range of applications in processing time sequence data. LSTM models proposed by Huang et al. [25] optimized past and future input features to enhance sequence tagging accuracy. Graves et al. [26] combined LSTM for speech recognition and achieved the best-recorded score to their knowledge. And Heigold et al. [27] proposed a novel end-to-end approach to speaker verification in LSTM, achieving better results.

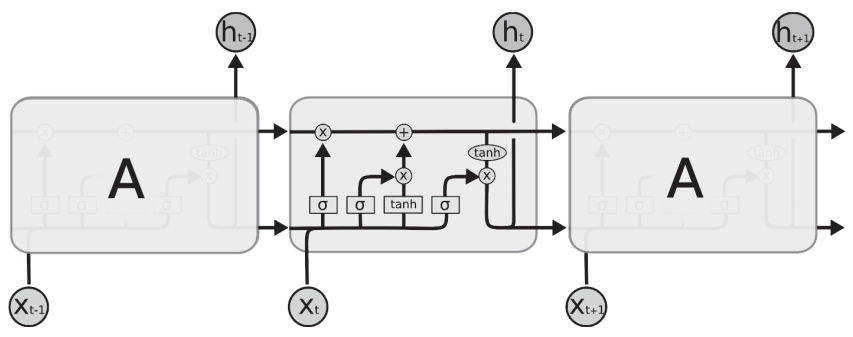

Fig. 1 Basic structure of LSTM [24]

LSTM offers powerful advantages of processing complex timeseries data and learning new features directly from raw data. In this study, we proposed to detect emotions based on fusion features of physiological signals - EEG and ECG signals - using ergonomically designed smart textile devices, and deep learning neural networks: LTSM. Our key aim is to develop a reliable alternative to traditional and expensive medical equipment for emotion detection of elderly people to facilitate the use of smart care in everyday care and applications; to improve the quality of care. Comfortable, wearable, low-cost and portable devices were developed for both EEG and ECG collection, allowing emotion detection on signals to be performed conveniently in everyday situations. We performed a prior experiment with healthy elderly people to monitor their EEG and ECG signals under different emotional states, then constructed deep learning models to classify emotions from raw signals directly. This paper is structured as follows: Section 2 discusses the design of the wearable textile devices: textile EEG cap and ECG chest band; Section 3 details the experiment and methods; Section 4 describes the data processing and classification results; Section 5 explains the findings. 


\section{Wearable textile devices}

\subsection{Textile EEG cap}

The conductive $\mathrm{Ag}-\mathrm{AgCl}$ coated dry EEG electrodes adopted were provided by OpenBCI Cooperation. The dry EEG electrodes were $3 \mathrm{D}$ printed with plastic substrate and coated with $\mathrm{Ag}-\mathrm{AgCl}$. The extended $(5 \mathrm{~mm})$ prongs of the electrodes could accommodate longer hair while enabling excellent signal quality. In our previous studies [17] we developed a 4-channel (F3*, F4*, P3*, and P4*) comfortable textile EEG cap with good signal quality for elderly people using ultra-soft gel electrode holders, as shown in Fig. 2 [17]. As discussed in the previous paper [17], the distribution and number of electrodes were determined based on emotion detection and wearing experience. While the frontal and parietal lobes have the largest amount of information on emotional states, excessive electrodes influence the perceived comfort of the users. The F3*, $\mathrm{F} 4 *, \mathrm{P} 3 *$, and $\mathrm{P} 4 *$ points (which are close to $\mathrm{F} 3, \mathrm{~F} 4, \mathrm{P} 3$, and $\mathrm{P} 4$ points) are distributed in the frontal and parietal cortex were selected as EEG channels in accordance with the International 10-20 system [28]. As shown in Fig. 2, the EEG electrode gel holder was made from urethane resin with a favorable ultra-soft and flexible design that maintains stable contact between the dry electrodes and the scalp without strong pressure. The blue protector used to separate the dry electrodes from the gel holder to support sound conductivity and electrode stability was made from plastic. In this study, we optimized the textile EEG cap with a beanie style design: to hide the wires, enhance stylistic features for a more natural appearance, and improve flexibility for added comfort and easy wear. The beanie cap is more friendly for elderly people and can be used as an accessory. We made beanie textile EEG caps in two sizes: M size for head circumference ranging from $(55 \sim 57) \pm 0.5$ $\mathrm{cm}$; L size for head size ranging from $(57 \sim 60) \pm 0.5 \mathrm{~cm}$.

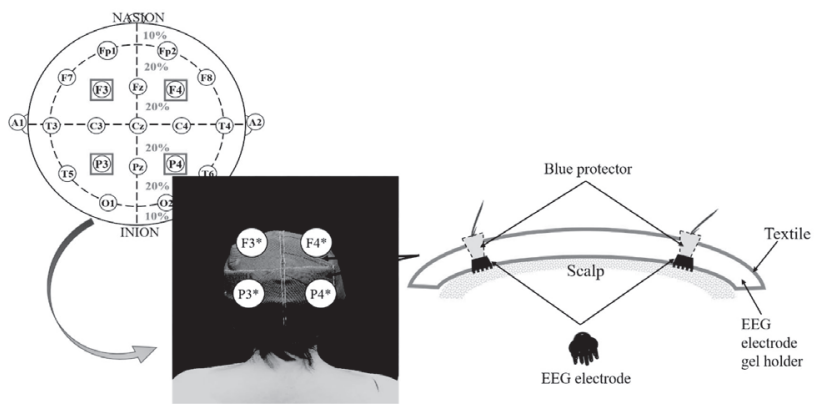

Fig. 2 Structure of the textile EEG cap with dry electrodes held by ultra-soft gel holders [17].

\subsection{ECG chest band}

For the ECG chest band, we utilized the conductive wearable knit fabric which features a nylon surface covered with a silvermetalized conductive fiber: AGposs ${ }^{\circledR}$ provided by Mitsufuji Corporation. The conductive fabric made of AGposs ${ }^{\circledR}$ is used as dry ECG electrode which offers enhanced electrical conductivity and flexible softness. To ensure enhanced and stable skin contact, the ECG electrode gel pads were introduced based on the same ideas and methods used to create an ultra-soft gel electrode holder for the EEG cap. The gel pad size was determined according to the size of the EEG signal sensor area. The central bio-sensing point was placed at the apex of the heart, which lies behind the fifth left intercostal space, $8-9 \mathrm{~cm}$ from the mid-sternal line. The main bio-sensing points were distributed around the apex of the heart; symmetrical to the mid-sternal line. While a wider sensing area offers better data acquisition, the larger the area the more chance of artifacts. The conductive knit fabric was therefore sized at $30 \mathrm{~mm} \times$ $40 \mathrm{~mm}$. As the gel pad needed to cover the sensing area - to increase the probability of the electrode to skin contact - we made the final version of the ECG gel electrode at $100 \mathrm{~mm}$ (long), $50 \mathrm{~mm}$ (wide), and $30 \mathrm{~mm}$ (high). An elastic band was made by sewing two pieces of the ground fabric made of rayon (R) $43 \%$, polyester (P) $51 \%$, polyurethane (PU) $6 \%$. Two conductive fabrics as ECG electrodes were then sewn on the band, with center points $9 \mathrm{~cm}$ away from the central line for the band. The gel pads were inserted into the band to cover the conductive fabric. Next, the snap buttons were punched into the conductive fabric on the band. A wearable chest band was fabricated for ECG collection in a comfortable and convenient way. We tested it on 15 people to compare the signal quality against the wet electrodes, getting a correlation coefficient of $94.2 \%$ with the wet electrodes. Fig. 3 shows the structure of the ECG chest band.

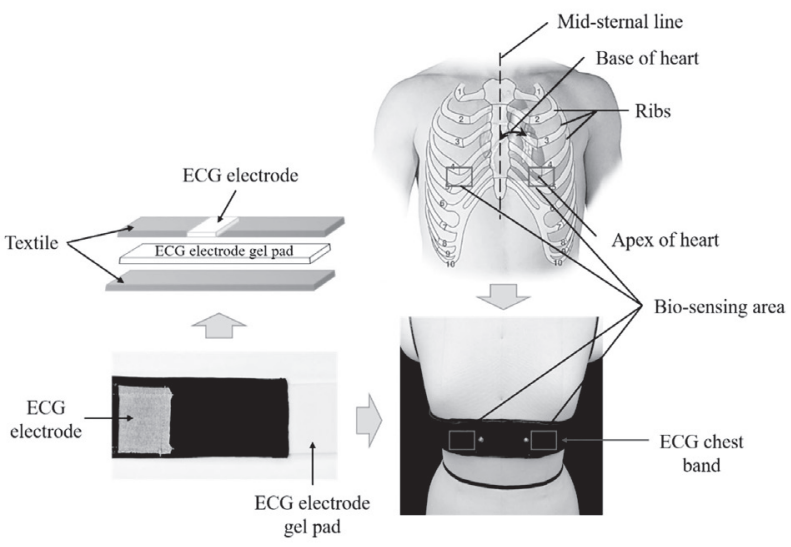

Fig. 3 Structure of the ECG chest band with conductive fabric using the ultra-soft gel pad.

\section{Method and experiment}

\subsection{Subjects and stimuli materials}

In this study, we invited 5 healthy elderly subjects to participate 
in this experiment: 2 women and 3 men, all aged over 65 years old $(\mathrm{M}=72.8, \mathrm{SD}=5.7)$ from Silver Human Resources Center, Kyoto, Japan. We selected elderly subjects due to the following reasons: (1) changes in CNS and ANS function are associated with normal healthy human ageing; (2) such changes due to ageing have led to an increased vulnerability and a decreased adaptation to environmental conditions, such as emotional stimulation; and (3) the attitude towards the physiological signals collecting devices tends to differ from that of younger subjects. The subjects were asked to read a detailed sheet containing general information and instructions about the experiment procedure in advance. A Japanese student of the team conducting the experiment was available to explain any questions posed by the Japanese subjects.

In the past, many techniques have been used for emotion elicitation, including the use of pictures, film-clips, imagination, and virtual reality. Compared to other methods, exposure to film-clips offers several advantages [29]. Firstly, it's one of the simplest techniques to conduct in a laboratory. Secondly, it is widely known that film can stimulate subjective and physiological changes to audiences. Thirdly, dynamic film scenes provide an artificial simulation of reality, which is thought to be the most powerful technique for eliciting emotions in laboratory conditions. In [30], the authors confirmed that physiological responses could be induced by emotion-eliciting films. We focused a lot of attention on searching and selecting appropriate stimuli films for elderly Japanese people. Previous studies showed that Western film-clips [31, 32] yielded consistent results in Japanese individuals at different ages. After discussing the suitability of the video for elderly people with Japanese professors and teachers, we selected 12 film-clips. The selection was based on film datasets [33, 34] designed to elicit four different emotions: happiness, anger, sadness, and fear ( 3 film-clips of about $1 \mathrm{~min}$ for each emotion). The film-datasets were summarized after several years of work by the researchers, which were also cited in studies by other researchers $[35,36,37]$. All the film-clips used in this experiment contain cut out scenes from different films, which are listed in Table 1.

Table 1 Detailed information of the utilized film-clips.

\begin{tabular}{c|cc}
\hline Emotion & Film & Lengths (s) \\
\hline Happiness & On Golden Pond & 39 \\
Happiness & Love Actually & 78 \\
Happiness & An Officer and a Gentleman & 65 \\
Anger & My Bodyguard & 54 \\
Anger & Witness & 59 \\
Anger & Gandhi & 53 \\
Sadness & The Champ & 59 \\
Sadness & The Killing Fields & 55 \\
Sadness & An Officer and a Gentleman & 37 \\
Fear & Marathon Man & 78 \\
Fear & Halloween & 75 \\
Fear & Silence of the Lambs & 52 \\
\hline
\end{tabular}

\subsection{Experiment protocol}

The experiments were conducted in a separate room with controlled lighting and conditions $\left(\mathrm{T}=25^{\circ} \mathrm{C}, \mathrm{RH}=50 \%\right)$ for comfort and to avoid external influences. We collected EEG and ECG signals on a PC equipped for recording (Dell Inspiron 7370). Stimuli videos were played on a Dell Alienware M15 PC. The subjects were seated approximately $70 \mathrm{~cm}$ from the screen. The speaker volume was set at a relatively loud level: Each subject was asked whether the volume was comfortable, and adjusted according to their preferences before starting the experiment. Each experiment for all the subjects were recorded with a video camera. EEG and ECG signals were collected simultaneously using our ergonomically designed 4-channel textile EEG cap (F3, F4, P3, P4) and ECG chest band with sampling at $250 \mathrm{~Hz}$. The ground electrodes were connected to the subject's ears. The data was collected using the Cyton bio-sensing board from OpenBCI platform and the signals were recorded and shown in real-time using OpenBCI GUI. Fig. 4 presents the scene of one subject during experiment.

The Japanese experimenter first explained the experiment process to the subjects clearly in Japanese. The subjects then signed a consent form stating that their physical information and recording images could be used for this study and not for other use. The head circumference of each subject was measured and fitted with the correct cap size. Next, the 4-channel textile EEG cap and ECG chest band were equipped to the subjects and the electrodes were placed in the right place.

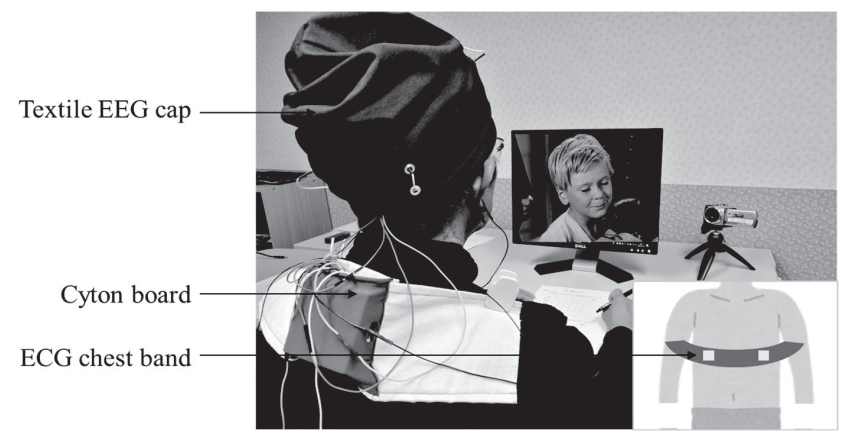

Fig. 4 One subject during the experiment.

The self-assessment form was explained to the subjects and they were asked to familiarize themselves with its contents. Before the formal experiment, the subjects were asked to sit comfortably way and remain as still as possible during experiment. We checked the stability of the signals and when the preparations were complete, commenced the experiment. Film-clips from all four categories (happiness, anger, sadness, fear) were played for the subjects at random; oscillating between each category (emotion). The formal experiment started with a 2 min of baseline recording while the subjects were asked to close their eyes. The 12 film-clips were 
played in 12 trials; taking about $30 \mathrm{~min}$ in total. A practice trial with a neutral state video was played for the subjects before watching the 12 film-clips to ensure the subjects thoroughly understood the experiment task. Each trial consisted of the following steps: (1) $15 \mathrm{~s}$ of quiet and recovery; (2) Screening of film-clips; (3) $30 \mathrm{~s}$ of self-assessment. During the experiment, each subject was asked to take a 5 min break after 6 th video to avoid fatigue. The whole experiment process is illustrated in Fig. 5.

After completing the experiment, we took off the devices from the subjects. When questioned, the subjects didn't complain of pain or discomfort caused by the devices when wearing them.

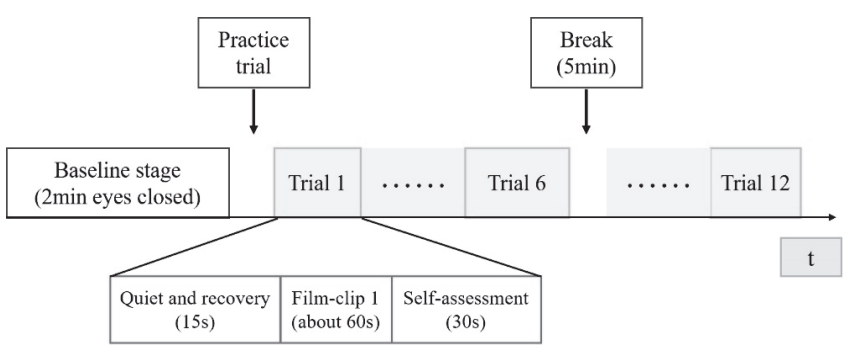

Fig. 5 Detail of the experiment processes and flow.

\subsection{Subjects self-assessment}

The subjects performed self-assessments to express their current emotion after watching film-clip in each trial. The subjects were asked to label the emotion felt while watching the stimuli filmclip (stimuli period) and rate how strongly they experienced it. They were not asked about the target emotion of the film-clip. We conducted a survey on the Self-Assessment Manikins (SAM) [38], often used by Japanese researchers and students, and found that SAM is difficult to understand for Japanese elderly people. We simplified the assessment form with each of the four emotion labels (happiness, anger, sadness, fear) scaled on a rating of 1 to $5(1=$ not at all, $5=$ very much). The assessment form was translated into Japanese.

The assessment data were analyzed to detect any variations on the subjects' assessment of the videos. The coefficient of variation (CV) of the subjects' assessment for all the film-clips was calculated. CV reflects the degree of data dispersion, which is defined as the ratio of the standard deviation of the original data to the average of the original data. High CVs indicates more variability. The mean CV between the subjects' rating for the happiness was $0.30 \pm 0.16$, for anger was $0.11 \pm 0.11$, for sadness was $0.23 \pm 0.13$, for fear was 0.20 \pm 0.12 , indicating a low variability between their assessments. All the ratings of each emotion for each video was 3 or over 3 . Our results show that the self-assessments of the subjects are consistent with the target emotions of the selected film-clips for this experiment.

\section{Results and discussion}

\subsection{Preprocessing}

All the saved .txt files were transferred to .csv format and the collected raw data of EEG and ECG was filtered with a Butterworth bandpass $(0.5 \sim 40 \mathrm{~Hz})$ to remove the main noises and artifacts to get the clean data. Then the EEG signals were then detrended using a Chebyshev I highpass filter to remove the baseline drift, and the ECG data de-trended using a Butterworth lowpass to remove baseline wander. The preprocessing method was performed by Python and SciPy, as illustrated in Fig. 6.
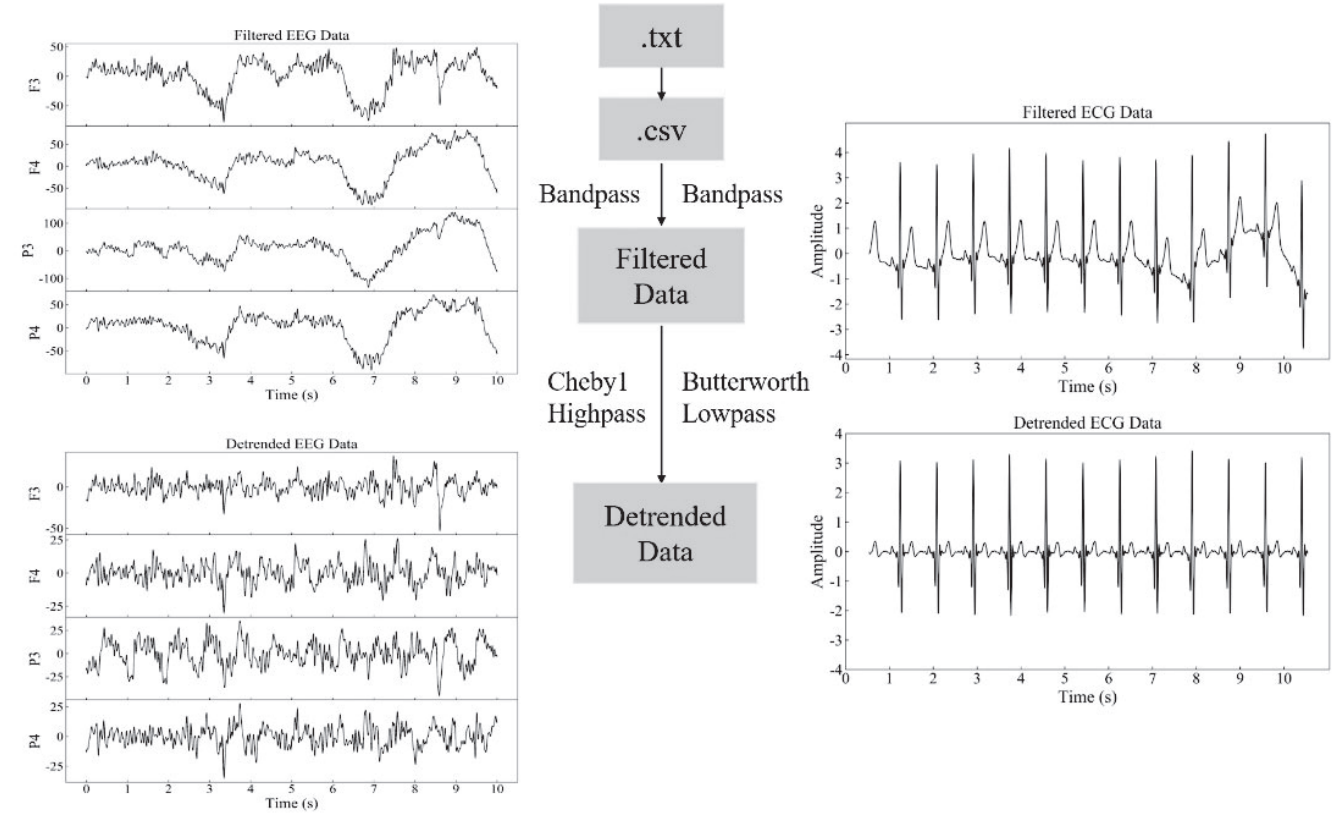

Fig. 6 Pre-processing steps of the raw data. 


\subsection{Dataset}

The samples from the five subjects were used to perform a binary classification of valence (positive/negative): Happiness was considered positive; Anger, sadness, and fear were considered negative. As three videos last just over thirty seconds, we assumed that the last 30 seconds recorded of EEG and ECG signals in the stimuli period (time period for watching the film-clip) would reflect the emotion of the subjects' ratings. When the rating score of the selected emotion was 3 or above 3 , this emotion is considered as the same as the target emotion. A total of 60 emotion samples of the five subjects distributed in positive (15 samples) emotion and negative (45 samples) emotion. The data file of each subject contains two arrays as shown in Table 2.

Table 2 Data arrays for each subject.

\begin{tabular}{c|cc}
\hline Array Name & Array Shape & Array Contents \\
\hline Data & $12 \times 5 \times 7500$ & Video $\times$ Channel $\times$ Data \\
Labels & $12 \times 4$ & $\begin{array}{c}\text { Video } \times \text { Label } \\
\text { (happiness, anger, sadness, fear) }\end{array}$ \\
\hline
\end{tabular}

As described in Table 2, each elderly subject has an array of 12 watched videos, 5 (EEG + ECG) channels, and 7500 readings. In this study, the EEG signals, only ECG signals, and EEG + ECG signals were studied separately.

\subsection{LSTM results}

In this study, we applied long short-term memory (LSTM) network to raw EEG, raw ECG, raw EEG + raw ECG signals of 5 subjects who watched the 12 film-clip to classify positive/negative valence. The deep learning neural network was conducted in Keras library with TensorFlow backend. There are researchers used LSTM for the time-series data and to process context information [39], which does not require any feature extraction. We designed LSTM1 for EEG data, LSTM2 for ECG data, and merged LSTM1 + LSTM2 for EEG + ECG data.

Emotional changes could be observed within seconds, even milliseconds of the EEG signal frame [40]. Therefore, to classify the emotions using EEG signals, we segmented each video sample of 4-channel EEG data into six 5-second segments. Each sample data consisted of 7500 readings for 4 EEG channels (F3, F4, P3 and P4) and a label. The LSTM1 designed for EEG data includes 3 LSTM layers to mine the context correlation in the input EEG feature sequence; 2 dropout layers with a probability of 0.2 to avoid overfitting; and a dense layer for integrating information for binary classification as shown in Fig. 7 (a). The first hidden layer contained 64 units; the second LSTM layer contained 32 units; the final LSTM layer contained 32 units. All the LSTM layers and the dense layer used sigmoid activation function.

Next, our researchers constructed the LSTM2 for ECG data. As the emotional changes could be observed in 3 15 seconds of the ECG signal frame [41], we segmented each video sample of ECG data into two 15-second segments. Each sample data consisted of 7500 readings for ECG data and a label. The LSTM2 used for ECG data includes 2 LSTM layers to mine the context correlation in the input ECG feature sequence, 1 dropout layer with a probability of 0.2 to avoid overfitting, and a dense layer for integrating information for binary classification as described in Fig. 7 (b). The first hidden layer contained 64 units, and the second LSTM layer contained 32 units. Both LSTM layers and the dense layer used sigmoid activation function.

Both of the two LSTM models used 4-fold cross-validation to ensure accuracy. 100 epochs were used in each irritation. For both models, the categorical-crossentropy was used as a loss function and stochastic gradient descent (SGD) optimizer was used with a 0.0001 learning rate. The average accuracy of LSTM1 with EEG data was $76.67 \%$, and the LSTM2 with ECG data was $75.00 \%$.

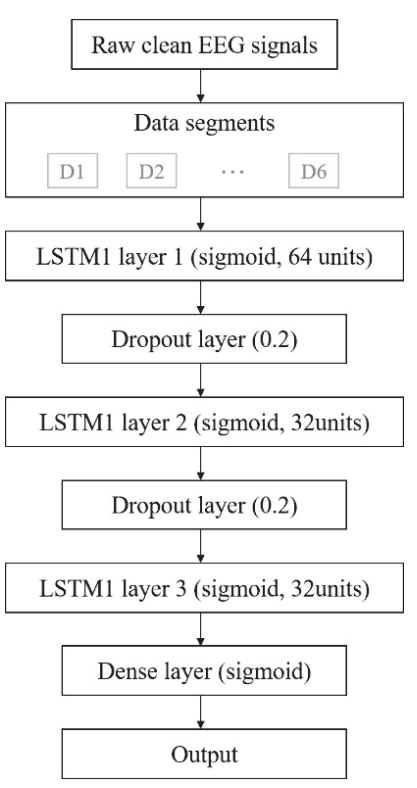

(a)

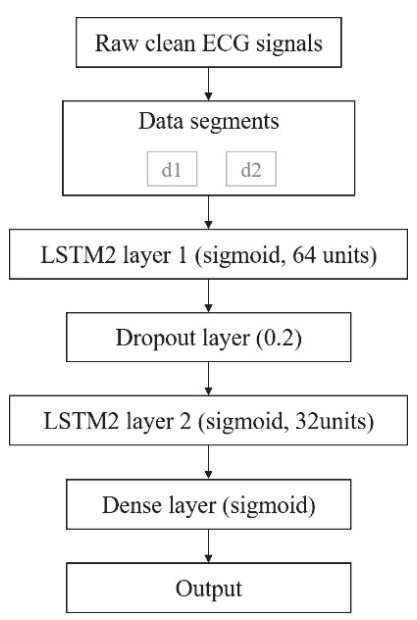

(b)
Fig. 7 Detailed view of LSTM models. (a) LSTM1; (b) LSTM2.

Finally, both 4-channel EEG data and ECG data were input into neural networks for the binary classification. To account for the variances in emotion exposure duration between EEG and ECG data, mentioned above, we segmented each video sample into three 10 -second segments. The values of EEG data and ECG data were normalized in the range of $(0,1)$ to compensate for the differences in the EEG and ECG signals. The "concatenate" function from "merge layers" in Keras was used to merge the two LSTM models, and the dense layer used the softmax activation function. The merged model 
also applied 4-fold cross-validation to obtain accuracy. 200 epochs were trained in each irritation. RMSprop optimizer was used for the merged model with a learning rate of 0.001 ; getting an accuracy of $95.00 \%$. Fig. 8 describes the processes of binary classification using EEG and ECG signals. The total trained parameters in the merged model was 3,266,574. Table 3 shows a complete comparison of accuracies between EEG, ECG, EEG and ECG.

We found that with the designed textile devices we could monitor EEG and ECG signals steadily, accurately and without causing discomfort to the wearer under the above experiment conditions. LSTM enabled us to process time-series data easily and effectively. As emotions are closely related to EEG (controlled by central nervous system), and ECG (controlled by autonomic nervous system), a combination of EEG and ECG signals greatly improved the emotion classification accuracy.

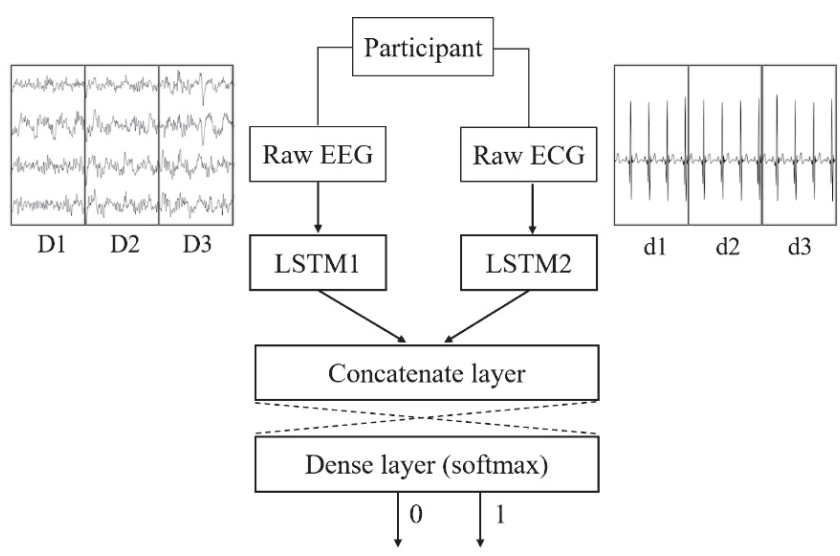

Fig. 8 Outline of the binary classification process of EEG and ECG signals.

Table 3 Comparison of accuracy results between EEG, ECG, EEG and ECG.

\begin{tabular}{c|cc}
\hline Signals & Models & Accuracy (\%) \\
\hline EEG & LSTM1 & 76.67 \\
ECG & LSTM2 & 75.00 \\
EEG + ECG & LSTM1 + LSTM2 & 95.00 \\
\hline
\end{tabular}

\subsection{Discussions}

We compared the best results obtained in this study with other recent related studies (Table 4). All of the works focused on valence classification: positive/negative emotion. Although the datasets of the works were different, it's clear that complex signals using EEG and ECG have a great contribution to emotion classification; which was consistent with our research. In [46], Shin.D et al achieved a higher accuracy of $98.06 \%$. This is due to several factors: (1) They used more electrodes for EEG and ECG, which may have caused subjects to feel unconformable; (2) the subjects were young, which means the physiological response may have been clearer compared to the elderly subjects; (3) more subjects participated in their experiment, which allowed them to obtain more samples. In this study, particular focus was placed on the comfort of the subjects during the experiment. However, the experiment results with 5 subjects may be too general for practical application. We therefore require more emotional data from more subjects to verify this method in future.

Table 4 A comparison of recent studies.

\begin{tabular}{c|ccc}
\hline Method & $\begin{array}{c}\text { Physiological } \\
\text { signals }\end{array}$ & Dataset & Accuracy (\%) \\
\hline Mohammadi Z (2017) [42] & EEG & DEAP & 86.75 \\
Alhagry S (2017) [43] & EEG & DEAP & 85.45 \\
Cheng Z (2017) [44] & ECG & Bio Vid Emo DB & 79.46 \\
Hsu YL (2017) [55] & ECG & 61 subjects & 82.78 \\
Shin D (2017) [46] & EEG + ECG & 30 subjects & 98.06 \\
Our approach & EEG + ECG & 5 subjects & 95.00 \\
\hline
\end{tabular}

\section{Conclusions and future work}

The purpose of the research in our team is to detect emotions for the elderly patients who lose speaking ability using smart textile devices in real-time with comfort and convenience. In our previous studies, constructed neural networks were used to detect emotional changes on arousal level using EEG and RRI spectrograms on young subjects [15]; collecting signals with wet electrodes. Next, we developed wearable textile devices (textile EEG cap and ECG chest band) for capturing EEG and ECG signals of elderly people with ease. The device was designed to fit like an everyday accessory item; paying attention to appearance and comfort [17]. In this study, we performed a prior experiment on healthy elderly subjects; monitoring their EEG and ECG signals through our designed textile devices under varying emotional states. To classify positive/negative emotions we applied raw signals (EEG; ECG; EEG + ECG) to end-to-end deep learning neural networks adopting LSTM to learn features. Our results show that combining EEG and ECG data to LSTM greatly improved the classification result, which is a very promising for emotion detection in future. The reasons for this are: LSTM has powerful ability to learn features from raw data directly; integrating EEG and ECG together provides more information for emotion detection; achieving high average accuracy over patients. The achievement of highly accurate on arousal [15] and valence classification in the study, suggests that emotion detection in dimensional emotion models, even discrete emotions, will yield good results.

The monitoring of the EEG and ECG signals for this study was conducted using our ergonomic textile EEG/ECG monitoring devices (described in Section 2) - designed for comfort, low-cost, portability - which would allow for the integration of emotion 
detection algorithms and smart textile devices into a range of applications such as elderly healthcare. The classification results using EEG and ECG signals was significantly more accurate than the results using single features. These findings further support our assumption that the use of low-cost, user-friendly and wearable textile devices for emotion detection is a reliable alternative to traditional and expensive medical equipment, and will facilitate the use of smart care to everyday care and application; improving the quality of care for elderly people. We intend to do further work to overcome the shortcomings of this paper. Firstly, a 5 subject sample group is too small to account for individual differences. Therefore, more data on emotions and EEG and ECG signals from a wider sample of healthy elderly people using our wearable textile devices are needed. Secondly, only a binary emotion classification on valence: positive/negative emotions was conducted in this study. Thus, we aim to implement the emotional physiological data to detect discrete emotions (happiness, anger, sadness, fear, etc.) using deep learning neural networks. This next step to realizing such a system of detecting emotions using wearable textile devices by deep learning in real-time has still not been solved with our present knowledge. Finally, we hope to put the device into practice with dementia patients to support their healthcare in the future.

\section{Acknowledgements}

This study was supported by the JSPS KAKENHI Grant Number 19H04154. We would also like to extend our appreciation to the China Scholarship Council (CSC) for supporting the Ph.D. research of Fangmeng ZENG, and to all the subjects from Silver Human Resources Center, Kyoto, Japan.

\section{References}

[1] Holden C (1987) Science, 236, 772-773. https://doi.org/ 10.1126/science. 3576194

[2] Stone R (2010) Science, 330, 1599. https://doi.org/10.1126/ science.330.6011.1599-a

[3] Sudo K, Kobayashi J, Noda S, Fukuda Y, Takahashi K (2018) Biosci Trends, 12, 7-11. https://doi.org/10.5582/bst.2017.01271

[4] Tsugane S (2020) European Journal of Clinical Nutrition. https://doi.org/10.1038/s41430-020-0677-5

[5] Muramatsu N (2016) "Developing Drug Products in an Aging Society", Chap 3, pp 45-57, Springer

[6] Vysata O, Kukal J, Prochazka A, Pazdera L, Simko J (2014) Neurologia I Neurochirurgia Polska, 48, 35-38. https://doi. org/10.1016/j.pjnns.2013.09.001

[7] Lipsitz LA, Novak V (2012) "Primer on the autonomic nervous system", Chap 271-273, Elsevier

[8] Berlin M, Wallace RB (1976) Experimental Aging Research, 2, 125-164. https://doi.org/10.1080/03610737608257984

[9] Dustman RE, LaMarche JA, Cohn NB, Shearer DE, Talone JM (1985) Neurobiology of Aging, 6, 193-198. https://doi. org/10.1016/0197-4580(85)90049-1
[10] Polich J (1997) Electroencephalography and Clinical Neurophysiology/Evoked Potentials Section, 104, 244-256. https://doi.org/10.1016/S0168-5597(97)96139-6

[11] Duffy FH, Albert MS, McAnulty G, Garvey AJ (1984) Annals of Neurology, 16, 430-438. https://doi.org/10.1002/ana.410160403

[12] Chadda KR, Ajijola OA, Vaseghi M, Shivkumar K, Huang CL-H (2018) Ageing Research Reviews, 48, 40-50. https://doi. org/10.1016/j.arr.2018.09.005

[13] Khane RS, Surdi AD, Bhatkar RS (2011) Journal of Basic and Clinical Physiology and Pharmacology, 22, 97-101. https://doi. org/10.1515/JBCPP.2011.017

[14] Wyss-Coray T (2016) Nature, 539, 180-186. https://doi. org/10.1038/nature20411

[15] Zeng FM, Liao PJ, Iwamoto M, Kuwahara N (2018) International Journal of Advanced Computer Science \& Applications, 9, 4954. https://doi.org/10.14569/IJACSA.2018.091006

[16] Kvajo M (2016) Cell, 167, 1443-1445. https://doi.org/10.1016/ j.cell.2016.11.029

[17] Zeng FM, Siriaraya P, Choi D, Kuwahara N (2020) International Journal of Advanced Computer Science \& Applications, 11(4), 58-66. https://doi.org/10.14569/ IJACSA.2020.0110409

[18] Atkinson J, Campos D (2015) Expert Systems with Applications, 47, 35-41. https://doi.org/10.1016/j.eswa.2015.10.049

[19] Jadhav N, Manthalkar R, Joshi Y (2017) Proceedings of International Conference on Computer Vision and Image, pp335-343

[20] Soleymani M, Lichtenauer J, Pun T, Pantic M (2012) IEEE Transactions on Affective Computing, 3, 42-55. https://doi. org/10.1109/T-AFFC.2011.25

[21] Guo HW, Huang YS, Lin CH, Chien JC, Shieh JS (2016) Proceedings of 2016 IEEE 16th International Conference on Bioinformatics and Bioengineering (BIBE)

[22] Valenza G, Citi L, Lanatá A, Scilingo EP, Barbieri R (2014) Scientific Reports, 4, 4998. https://doi.org/10.1038/srep04998

[23] Kim J, André E (2008) IEEE Transactions on Pattern Analysis and Machine Intelligence, 30, 2067-2083. https://doi. org/10.1109/TPAMI.2008.26

[24] Hochreiter S, Schmidhuber J (1997) Neural Computation, 9, 1735-1780. https://doi.org/10.1162/neco.1997.9.8.1735

[25] Huang Z, Xu W, Yu K (2015) arXiv preprint arXiv:1508.01991

[26] Graves A, Mohamed A, Hinton G (2013) Proceedings of 2013 IEEE International Conference on Acoustics, Speech and Signal Processing (ICASSP), 6645-6649

[27] Heigold G, Moreno I, Bengio S, Shazeer N (2016) Proceedings of 2016 IEEE International Conference on Acoustics, Speech and Signal Processing (ICASSP), 5115-5119

[28] Technologies TC (2012) "10/20 System Positioning Manual", Trans Cranial Technologies New York, NY

[29] Schaefer A, Nils F, Sanchez X, Philippot P (2010) Cognition and Emotion, 24, 1153-1172. https://doi. 
org/10.1080/02699930903274322

[30] Fernández C, Pascual JC, Soler J, Elices M, Portella MJ, Fernández-Abascal E (2012) Applied Psychophysiology and Biofeedback, 37, 73-79. https://doi.org/10.1007/s10484-0129180-7

[31] FakhrHosseini SM, Jeon M (2017) "Emotions and Affect in Human Factors and Human-Computer Interaction", Chap 10, pp235-253, Academic Press

[32] Sato W, Noguchi M, Yoshikawa S (2007) Social Behavior and Personality: an international journal, 35, 863-874. https://doi. org/10.2224/sbp.2007.35.7.863

[33] Hewig J, Hagemann D, Seifert J, Gollwitzer M, Naumann E, Bartussek D (2005) Cognition and Emotion, 19, 1095-1109. https://doi.org/10.1080/02699930541000084

[34] Gilman TL, Shaheen R, Nylocks KM, Halachoff D, Chapman J, Flynn JJ, Matt LM, Coifman KG (2017) Behavior Research Methods, 49, 2061-2082. https://doi.org/10.3758/s13428-0160842-x

[35] Wang XW, Nie D, Lu BL (2014) Neurocomputing, 129, 94106. https://doi.org/10.1016/j.neucom.2013.06.046

[36] Codispoti M, Surcinelli P, Baldaro B (2008) International Journal of Psychophysiology, 69, 90-95. https://doi. org/10.1016/j.ijpsycho.2008.03.004

[37] Moreira PS, Chaves P, Dias N, Costa P, Almeida PR (2018) PsyArXiv. https://doi.org/10.31234/osf.io/kmpq5
[38] Backs RW, da Silva SP, Han K (2005) Experimental Aging Research, 31, 421-440. https://doi.org/10.1080/ 03610730500206808

[39] Li Y, Huang J, Zhou H, Zhong N (2017) Applied Sciences, 7, 1060. https://doi.org/10.3390/app7101060

[40] Codispoti M, Mazzetti M, Bradley MM (2009) Psychophysiology, 46, 731-738. https://doi.org/10.1111/j.14698986.2009.00804.x

[41] Dissanayake T, Rajapaksha Y, Ragel R, Nawinne I (2019) Sensors, 19, 4495. https://doi.org/10.3390/s19204495

[42] Mohammadi Z, Frounchi J, Amiri M (2017) Neural Computing and Applications, 28, 1985-1990. https://doi.org/10.1007/ s00521-015-2149-8

[43] Alhagry S, Fahmy AA, El-Khoribi RA (2017) International Journal of Advanced Computer Science and Applications, 8, 355-358. https://doi.org/10.14569/IJACSA.2017.081046

[44] Cheng Z, Shu L, Xie J, Chen CLP (2017) Proceedings of 2017 International Conference on Security, Pattern Analysis, and Cybernetics (SPAC), 296-301

[45] Hsu Y, Wang J, Chiang W, Hung C (2017) IEEE Transactions on Affective Computing, 11, 85-99. https://doi.org/10.1109/ TAFFC.2017.2781732

[46] Shin D, Shin D, Shin D (2017) Multimedia Tools and Applications, 76, 11449-11470. https://doi.org/10.1007/ s11042-016-4203-7 